\title{
The Education and Professional Standards Committee (EPSC)
}

\begin{abstract}
Ultrasound is an effective imaging modality used worldwide for clinical work-up of patients with a wide range of diseases, particularly as the first line imaging modality. Technological progress has opened new perspectives to ultrasound (US) clinical applications extending its use with increased diagnostic efficacy. Therefore, education and subsequently preparing and updating of US knowledge is crucial. New technologies and web-based sources of information have opened even more and reliable educational applications in medical practice.
\end{abstract}

Therefore, in the last years EFSUMB (European Federation of Societies for Ultrasound in Medicine and Biology) Education (EPSC), Publication and Safety (ECMUS) committees have worked together to promote ultrasound knowledge in all the European countries.

Among the most significant activities to be mentioned, is the EFSUMB website (www.efsumb.org) recently renovated, and enriched with different sections; (1) "Case of the month" section has regular contributions of interesting cases and translated into 15 languages. (2) First webinar on CEUS for liver application that has been successfully promoted and launched by the EPSC in December 2015 is freely available on the website with full video-recorded material for viewing and download. Almost 2 hundred colleagues were connected in streaming, following the lectures which were held by recognized experts in CEUS liver evaluation as Prof. Christoph Dietrich (Germany), Prof. Odd Helge Gilja (Norway), and Prof. Fabio Piscaglia (Italy). The attendees after any lecture participated in discussion, which was addressed by the chairman and experts. EFSUMB is preparing future webinars on other applications of CEUS and US elastography and they will be launched in the next months.

Furthermore, one of the main activities in the last and next years of EFSUMB and EPSC is Medical student education in ultrasound. To this end the EFSUMB Course Book (ECB), EFSUMB Course Book Student Edition (ECBSE), eBooks, an Atlas of images including echoscopy and teaching videos (anatomy, examination technique) are available online and free to download from the EFSUMB website (www.efsumb. org). EFSUMB has decided to establish a separate Student Committee in the federation that will promote student education and relevant activities in Europe. EFSUMB is recruiting students from different European countries to form an interim board. Two EFSUMB position papers have been recently published in UiM/EJU (short version) and UIO (long version) (free for download). Furthermore, EFSUMB plan to integrate a new Student Congress within future EUROSON annual congresses. A survey on the present status of Ultrasound teaching during medical School training has been prepared by the EPSC. Period, length, facilities, activities, curriculum used to achieve this goal will be asked to deans of universities and representatives of the student organizations in order to establish the present status.

Universities will be requested to provide ideas and present limitations and difficulties to implement ultrasound as a teaching instrument from the start of new medical doctors' preparation.

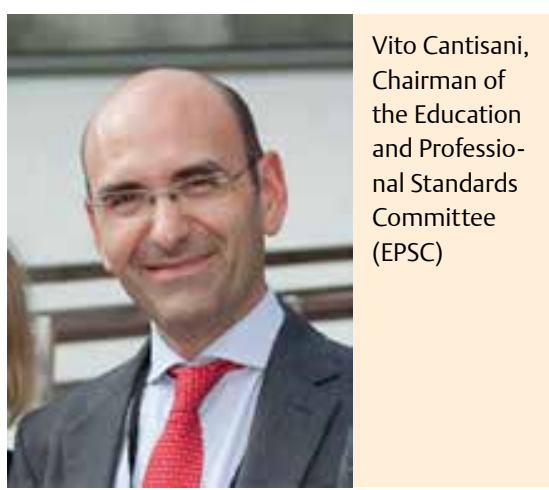

An official letter will follow up to the different universities who respond and to all the National Universities of the student representative organizations about the proposal of EFSUMB for the inclusion of US in the medical education core curriculum.

One-year prospective integration of anatomy and physiology teaching by means of US in 4-6 selected universities will be launched to develop an appendix of core curriculum.

In the next EUROSON, which will be held in Leipzig 26-29 October 2016, the 3 committees will organize a joint session to discuss the first results on activities for student ultrasound medical education implementation.

\section{Dr Vito Cantisani \\ Chairman EPSC \\ vito.cantisani@uniroma1.it \\ Department of Radiological Sciences \\ University Sapienza \\ Rome Italy}

